\title{
Is avoiding full sib matings really worthwhile in a breeding program?
}

\author{
Mathilde Dupont-Nivet ${ }^{\mathbf{a},{ }^{*}}$ and Marc Vandeputte ${ }^{\mathbf{a}, \mathbf{b}}$
}

a INRA, UMR1313 Unité de Génétique Animale et Biologie Intégrative, F-78350 Jouy-en-Josas, France

${ }^{\mathrm{b}}$ Ifremer, Chemin de Maguelone, F-34250 Palavas-les-Flots, France

* Corresponding author : Mathilde Dupont-Nivet, Tel.: + 331346523 49; fax: + 331346523 90, email address: mathilde.dupont-nivet@jouy.inra.fr

\begin{abstract}
:
Stochastic simulations were used to compare genetic progress and inbreeding depending on whether full sib matings were allowed or not when applying individual selection. Fifteen selection cycles were considered, using a single pair mating design. Two levels of heritability $\left(h^{2}\right)$ were studied $(0.1 ; 0.5)$ as well as four different proportions of selected animals (2, 5, 10 and 20\%), four population sizes (1000, $2,000,4,000$ and 10,000 candidates) and two levels of inbreeding depression ( 0 and $5 \%$ decrease of phenotype for $10 \%$ increase in inbreeding). Fifty replicates were generated for each set of parameters. The simulations revealed that avoiding full sib matings became less and less relevant as population size and proportion of selected animals increased and as heritability and impact of inbreeding on phenotype decreased. In large populations (10,000 candidates), excluding full sib matings was not worthwhile at all whereas in small populations with high selection intensity, it was a first useful way to manage genetic variability. This can be illustrated in two extreme situations. With 1000 candidates, $\mathrm{h}^{2}=0.5,5 \%$ inbreeding depression and $2 \%$ selection pressure, the phenotypic mean after 15 generations of selection was $18.9 \%$ greater when full-sib matings were excluded rather than allowed, while inbreeding was $33 \%$ lower. Conversely, for a large population (10,000 candidates), when the proportion of selected animals was over $5 \%$, there was no significant difference in phenotypic mean after 15 generations of selection whether excluding full-sib matings or not, while the difference in inbreeding was below $0.07 \%$ per generation.
\end{abstract}

Keywords: Selection; Sib mating; Inbreeding; Genetic variability; Stochastic simulations; Fish 


\section{Introduction}

When managing a selective breeding program there should be a balance between making as much genetic progress as possible in the short run and keeping high genetic variability for future selection. The most important point is the management of selection which has a major impact on preservation of genetic variability. Excessive culling increases inbreeding and reduces genetic variability (e.g., Bentsen and Olesen, 2002), and is always a danger in fish breeding programs, as the high fertility and generally small size of breeding candidates makes very high selection intensities potentially feasible. Once selection has been carried out, the mating strategy also has an impact on the preservation of genetic variability. Many studies have investigated different mating strategies: minimum coancestry, compensatory matings (Caballero et al., 1996), circular matings (de Rochambeau and Chevalet, 1985) among others. Methods combining selection choice and mating strategies have also been proposed: optimum contribution selection methods for maximising genetic level with constrained inbreeding (Sonesson and Meuwissen, 2000), dynamic minimization of the average pairwise kinship coefficient (Colleau et al., 2004). All these methods have been proven to be efficient. However, their implementation requires specific knowledge and computational skills, and thus they are not accessible to every breeder on the field. Simple ways for preserving genetic variability are generally taught to breeders: maintaining high effective population size by using a high number of parents (Bentsen and Olesen, 2002), balancing number of sires and dams as well as number of offspring from each family (Chevassus, 1989). Finally, mating related animals is often feared by breeders and many of them manage their breeding scheme with exclusion of full sib matings as advised in many scientific papers (for example, Waples and Do, 1994; Sekino et al., 2004; Hansen and Jensen, 2005). However, it is not obvious whether this exclusion is really useful or is only a generally accepted idea. Indeed, exclusion of full sib matings will of course reduce the inbreeding level at the next generation, but not totally since it does not exclude the mating of more distant relatives. Moreover, the inbreeding created by full sib matings at the next generation will probably be diluted at further generations as inbred animals will be mated with unrelated animals if the population is large enough. Moreover, excluding full sib matings has a significant cost in fish individual breeding programs, as hatchlings are too small to be tagged and pedigrees must be recovered using genetic markers (Estoup et al, 1998) or separate rearing of families until tagging size.

The aim of the present paper was to study the usefulness of the simple exclusion of full sib matings in individual breeding programs. Stochastic simulations were used to estimate the effect of allowing or not full sib matings in an individual breeding program, with a single pair mating design, considering different levels of heritability, proportion of selected animals, number of breeding candidates, and inbreeding depression.

\section{Material and methods}

\subsection{Simulation program}

A computer program, written in Fortran 90 (using NAG Fortran Library), was used to simulate individual selection. The evolution of a quantitative trait was simulated according to a polygenic model. Genetic values of founders were randomly drawn from a normal distribution $\mathrm{N}\left(0, \sigma^{2}{ }_{a 0}\right)$, where $\sigma^{2}{ }_{a 0}$ is the additive genetic variance in the base population. At each generation, the phenotypic value of each offspring was calculated from the additive genetic values of its parents, a term accounting for Mendelian sampling and a random environmental effect drawn from a normal distribution $\mathrm{N}\left(0, \sigma^{2}\right)$. The Mendelian sampling term was randomly drawn from a normal distribution $\left(\mathrm{N}\left(0, \frac{\sigma^{2} a 0}{2}\left(1-\frac{F s+F d}{2}\right)\right)\right.$, where $\mathrm{Fs}$ and Fd were the 
inbreeding coefficients of the sire and the dam, respectively. Other details are available in Dupont-Nivet et al. (2006).

The inbreeding coefficient was calculated using a subroutine written by Boichard (Boichard et al., 1997), based on the method of Meuwissen and Luo (1992). A direct effect of inbreeding on the phenotype was simulated considering a linear decrease in performance as inbreeding increased, as observed in many data sets (Lynch and Walsh, 1998).

Sex was randomly attributed to the offspring, assuming each sex had equal probability. Then animals from each sex were sorted according to their phenotypic value and the ones with highest values kept for mating. Animals were mated according to a single pair mating design: $\mathrm{s}$ sires were mated to $\mathrm{s}$ dams, in such a way that one sire was mated to only one dam, and each dam was mated to only one sire, producing s full sib families. Mating between full sibs could be either avoided (NFS) or allowed (FS).

The selection process described above was carried out for 15 generations, and was repeated 50 times, and the mean inbreeding and phenotypic mean were calculated.

\subsection{Parameters studied}

Different combinations of parameters were used in order to study a range of situations:

The total population size, before selection, was set to 1,$000 ; 2,000 ; 4,000$ and 10,000 animals that were assumed to be sexually mature (and thus breeding candidates). No mortality was considered so that family sizes were equal before selection.

The number of selected sires and dams were chosen so that 4 levels of proportion of selected animals could be studied: 2, 5, 10 and 20\%. This means that the number of full sib families was 10, 25, 50 or 100 when the total population total size was 1,000 and 100, 250, 500 and 1000 for the largest population total size $(10,000)$.

Initial genetic variance, $\sigma^{2}$ ao and environmental variance, $\sigma^{2}{ }_{\mathrm{e}}$ were set so that two levels of heritability (0.1 and 0.5) could be considered, whereas $\sigma^{2}{ }_{a 0}+\sigma^{2} \mathrm{e}$ was kept constant in the base population at an arbitrary value of 20 . Finally two levels of direct effect of inbreeding on phenotype (DecF) were considered: 0 and $5 \%$ decrease in performance for a $10 \%$ increase in inbreeding.

\section{Results and discussion}

Phenotypic means of the population (P) after 15 generations of selection for different population sizes $(\mathrm{N})$ and different proportions of selected animals $(\mathrm{pr})$ are reported in Figure 1 for $h^{2}=0.5$ (a) and $h^{2}=0.1$ (b). When population was the smallest $(\mathrm{N}=1000)$ and selection was the most intense (2\%), it was not possible to run a selection over 15 generations avoiding full sib matings. Indeed, the number of breeders at each generation was small (20, i.e. 10 sires and 10 dams), thus there was always one generation for which it became impossible to mate animals which were not full sibs. In Table 1, standard errors of phenotypic mean for each combination of parameters, calculated from the fifty replicates are given as well as significance of differences between phenotypic means (reported in figure 1) between NFS and FS, tested with a t-test.

Figure 1 shows that the effect of avoiding full sib matings increased when decF or heritability, or both, increased and when population size or proportion of selected animals decreased, or both. For large populations $(\mathrm{N}=10,000)$, the difference between NFS and FS was significant only for $\mathrm{pr}=2 \%$ and $\mathrm{h}^{2}=0.5$. However, even if significant, differences were small, the phenotypic mean for NFS after 15 generations being always less than $1.5 \%$ greater than that for FS. For small populations, the differences were much larger. For example, the phenotypic mean was $18.9 \%$ greater for NFS (compared to FS) when $N=1,000, h^{2}=0.5$, decF $=5$ and $\mathrm{pr}=2 \%$, which is a scheme with very efficient short term selection. This difference declined rapidly when decF decreased: it was $+5.9 \%$ only when decF $=0$. 
Inbreeding $(F)$ is reported in Figures $2 \mathrm{a}$ and $2 \mathrm{~b}$. Results were consistent with those for phenotypic means. Due to the low variation of $F$ between replicates, differences between NFS and FS were almost always significant $(P<0.05$ except for $\mathrm{N}=10,000$ and $\mathrm{pr}=2 \%$, and for $\mathrm{N}=1,000, \mathrm{pr}=10 \%, \mathrm{~h}^{2}=0.1$ and $\operatorname{dec} \mathrm{F}=0$ ). The highest difference was for $\mathrm{N}=1,000, \mathrm{pr}=2 \%$, $\mathrm{h}^{2}=0.5$ and $\operatorname{dec} \mathrm{F}=5 \%$, in which case $\mathrm{F}$ was $33.3 \%$ greater when full sib matings were allowed, corresponding to an absolute increase in $\mathrm{F}$ of 0.148 (or 0.01 per generation). For large populations $(\mathrm{N}=10,000)$ absolute differences in $\mathrm{F}$ were always lower than $0.01(0.0007$ per generation) whatever the other parameters.

The evolution of phenotypic mean and inbreeding with level of inbreeding depression, population size, heritability and proportion of selected animals were quite logical. Indeed, when heritability increases, selected animals will originate from the same better performing families, thus unbalancing founder representation and increasing inbreeding level through the selection of more related animals. More intense selection will obviously also increase the probability to select related animals. This trend will be exhacerbated in small populations, and also when decF $=5 \%$ because in this case inbred animals will have a lower probability to be selected thus further unbalancing the representation of founders. By contrast, for large populations and less efficient short term selection, the probability of selecting related animals is lower. Moreover, even if some related animals are mated, this will have little impact, first because it will only concern a few matings among a large number, and also because offspring of full sib matings have a low probability to mate again with related animals.

Our results are consistent with literature results. However, results in the literature concern only small size populations. Caballero et al. (1996) found that avoidance of full sibs was better (especially in terms of final inbreeding) than random mating. They also showed that avoidance of full sib matings was more useful with high rates of inbreeding caused by BLUP selection or small population size which is again consistent with our results. Wang (1997) showed that avoidance of full sib matings is the most useful with equal numbers of sires and dams. He also showed that when number of sires and dams increase, the effect of sib mating avoidance decreases. We must underline also that in these papers, the effect of inbreeding depression was not taken into account as it is in our simulations.

As a conclusion, in fish species for which the number of breeding candidates is usually high and pedigree knowledge is expensive (requiring either separate rearing of families or genotyping of candidates), different cases must be considered.

In species for which artificial selection is very well mastered like salmonids and for which initial population sizes are large (at least ten thousand animals at young stages before selection) exclusion of full sib matings is not useful, as long as selection is reasonably managed with enough breeders at each generation and without excessive culling.

By contrast, in some species it can be difficult to have large initial population size and large number of breeders, because of technical or economical problems. We considered in our paper that it was easy to obtain sexually mature animals ready to reproduce which is not always obvious. For example, in seabass, it is highly technical to obtain more than 10 dams spawning synchronously (Chatain, pers. comm.). We also considered pair matings and supposed all breeders participate to reproduction and all of them have high reproductive rates. This is not always realistic: in mass spawnings, for example in tilapia, sea bass or sole, the number of effective breeders is always lower than expected (Fessehaye et al., 2006; Chatziplis et al., 2007; Blonk et al., 2009). In these cases, avoiding full sib matings can be helpful to obtain higher genetic response and to reduce the increase in inbreeding. However, once the genetic variability is lost, the mating strategies will only delay the problem and thus, the most important thing would be to make all efforts to obtain enough effective breeders. Fish are very prolific species and temptation can be high to use very few breeders to renew the stock. It is essential to avoid this temptation. The survey about selection practices published by the Aquabreeding network (2009) showed that many European breeders are aware of that since, out of 35 selected lines, $83 \%$ are maintained with more than 100 breeders.

Finally it must be noted that avoidance of full sib matings is simple to implement (assuming pedigrees are available) but it will always be more efficient in the long term to acquire skills to 
use more advanced methods for optimizing genetic progress together with inbreeding. Possible solutions are either optimum contribution selection methods for maximizing genetic level with constrained inbreeding (Gjerde et al., 1996; Villuaneva et al., 1996), either dynamic minimization of the average pairwise kinship coefficient (Colleau et al., 2004),

\section{References}

AquaBreeding survey: Survey on the Breeding Practices in the European aquaculture industry, $2009 . \quad$ [Online]. Available: http://www.aquabreeding.eu/Documents/tabid/98/Default.aspx.

Bentsen, H.B., Olesen, I., 2002. Designing aquaculture mass selection programs to avoid high inbreeding rates. Aquaculture 204, 349-359.

Blonk, R.J.W., Komen, J., Kamstra, A., Crooijmans, R.P.M.A., van Arendonk, J.A.M., 2009. Levels of inbreeding in group mating captive broodstock populations of Common sole, (Solea solea), inferred from parental relatedness and contribution. Aquaculture 289, 26-31.

Boichard, D., Maignel, L., Verrier, E., 1997. The value of using probabilities of gene origin to measure genetic variability in a population. Genet. Sel. Evol. 29, 5-23.

Caballero, A., Santiago, E., Toro, M.A., 1996. Systems of mating to reduce inbreeding in selected populations. Anim. Sci. 62, 431-442.

Chatziplis, D., Batargias, C., Tsigenopoulos, C.S., Magoulas, A., Kollias, S., Kotoulas, G., Volckaert, F.A.M., Haley, C.S., 2007. Mapping quantitative trait loci in European sea bass (Dicentrarchus labrax): The BASSMAP pilot study. Aquaculture 272, S172-S182

Chevassus, B., 1989. Aspects génétiques de la constitution de populations d'élevage destinées au repeuplement. Bull. Fr Pêche Pisc. 314, 146-168.

Colleau, J.J., Moureaux, S., Briend, M., Bechu, J., 2004. A method for the dynamic management of genetic variability in dairy cattle. Genet. Sel. Evol. 36, 373-394.

Dupont-Nivet, M., Vandeputte, M., Haffray, P., Chevassus, B., 2006. Effect of different mating designs on inbreeding, genetic variance and response to selection when applying individual selection in fish breeding programs. Aquaculture 252, 161-170

Estoup, A., Gharbi, K., SanCristobal, M., Chevalet, C., Haffray, P., Guyomard, R., 1998. Parentage assignment using microsatellites in turbot (Scophthalmus maximus) and rainbow trout (Oncorhynchus mykiss) hatchery populations. Can. J. Fish. Aqua. Sci. 55, 715-725.

Fessehaye, Y., El-bialy, Z., Rezk, M.A., Crooijmans, R., Bovenhuis, H., Komen, H., 2006. Mating systems and male reproductive success in Nile tilapia (Oreochromis niloticus) in breeding hapas: a microsatellite analysis. Aquaculture 256, 148-158.

Gjerde, B., Gjøen, H.M., Villanueva, B., 1996. Optimum designs for fish breeding programmes with constrained inbreeding. Mass selection for a normally distributed trait. Livest. Prod. Sci. 47, 59-72.

Hansen, M.M., Jensen, L.F., 2005. Sibship within samples of brown trout (Salmo trutta) and implications for supportive breeding. Cons. Genet. 6, 297-305.

Lynch, M., Walsh, B., 1998. Genetics and analysis of quantitative traits. Sinauer Assoc., USA.

Meuwissen, T.H.E., Luo Z., 1992. Computing inbreeding coefficients in large populations. Genet. Sel. Evol. 24, 305-313.

Rochambeau, H. de, Chevalet, C., 1985. Minimisation of the average inbreeding coefficient in small populations of domestic animals. Genet. Sel. Evol., 17, 459-480

Rochambeau, H. de, Fournet-Hanocq, F., Khang, J.V.T, 2000. Measuring and managing genetic variability in small populations. Ann. Zoot. 49, 77-93.

Sekino, M., Sugaya, T., Hara M., Taniguchi, N., 2004. Relatedness inferred from microsatellite genotypes as a tool for broodstock management of Japanese flounder Paralichthys olivaceus. Aquaculture 233, 163-172.

Sonesson, A.K., Meuwissen, T.H.E., 2000. Mating schemes for optimum contribution selection with constrained rates of inbreeding. Genet. Sel. Evol. 32, 231-248. 
Villanueva, B., Woolliams, J.A., Gjerde, B., 1996. Optimum designs for breeding programmes under mass selection with an application to fish breeding. Anim. Sci. 63, 563576.

Wang, J., 1997. Effect of excluding sib matings on inbreeding coefficient and effective size of finite diploid populations. Biometrics 53, 1354-1365.

Waples, R.S., Do, C., 1994. Genetic risk associated with supplementation of pacific Salmonids: captive broodstock programs. Can. J. Fish. Aquat. Sci. 51 (suppl. 1), 310-329.

\section{Figures}

Figure 1 - Phenotypic mean (P) after 15 individual selection cycles, for different values of population size before selection, proportion of selected animals, and decrease of phenotype with inbreeding (decF), with full sib matings allowed (FS) or avoided (NFS), for $h^{2}=0.5$ (a) and $h^{2}=0.1(b)$

Figure 2 - Inbreeding coefficient (F) after 15 individual selection cycles, for different values of population size before selection, proportion of selected animals, and decrease of phenotype with inbreeding (decF), with full sib matings allowed (FS) or avoided (NFS), for $\mathrm{h}^{2}=0.5$ (a) and $h^{2}=0.1(b)$ 
Fig. 1

0

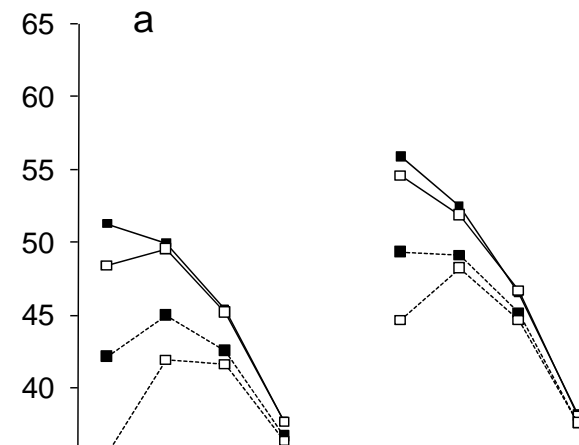

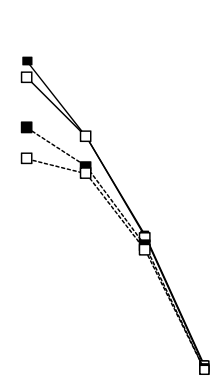

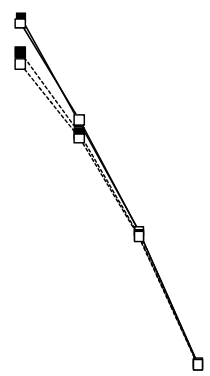

$h^{2}=0.5$

$\rightarrow-F S$, decF $=0$

- NFS, decF $=5$

$\cdots \cdot \cdots \mathrm{FS}, \mathrm{dec} F=5$

30

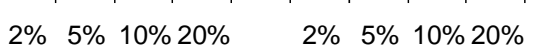

$2 \% \quad 5 \% \quad 10 \% 20 \%$

$2 \% \quad 5 \% \quad 10 \% 20 \% \quad \%$ of selected animals

1000
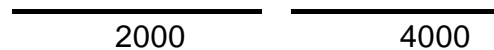

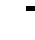

10000

Population size

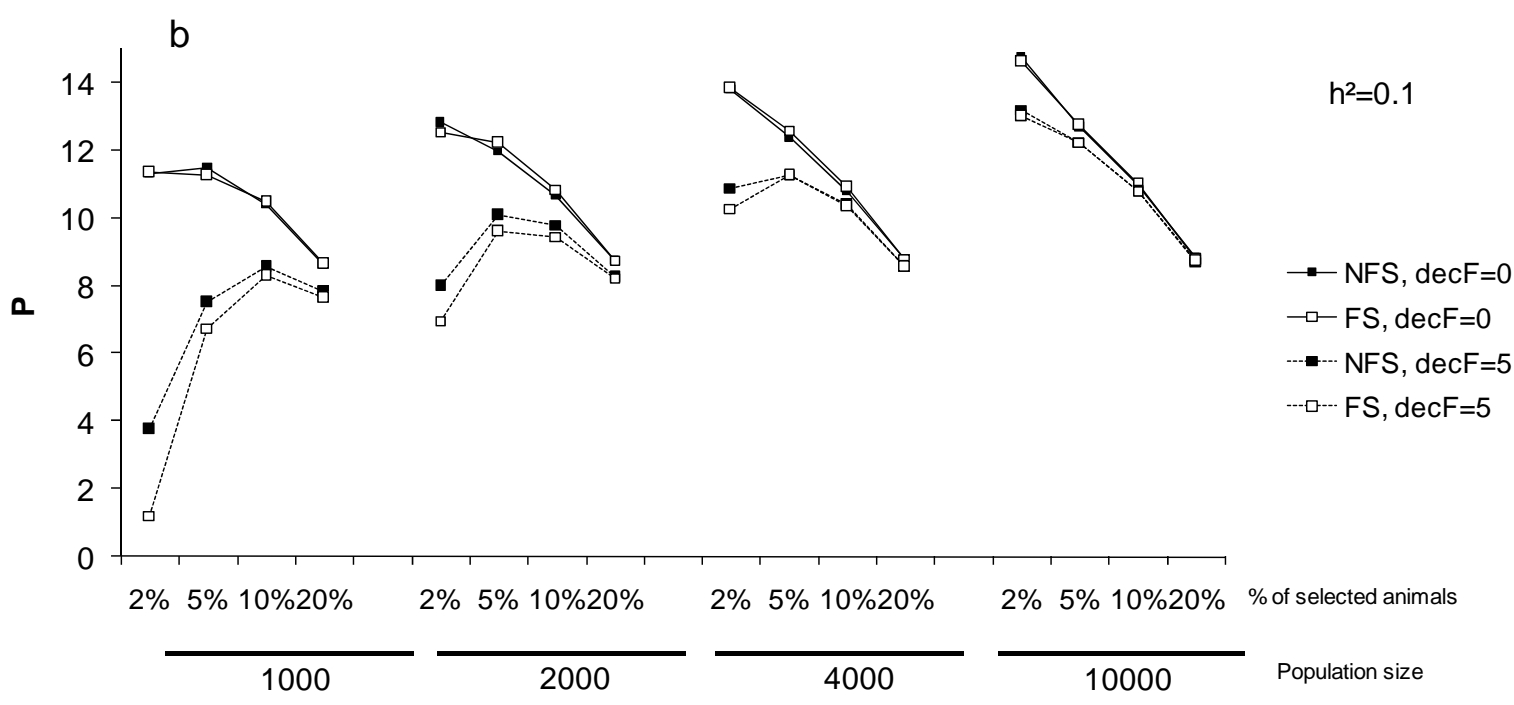


Fig. 2

a

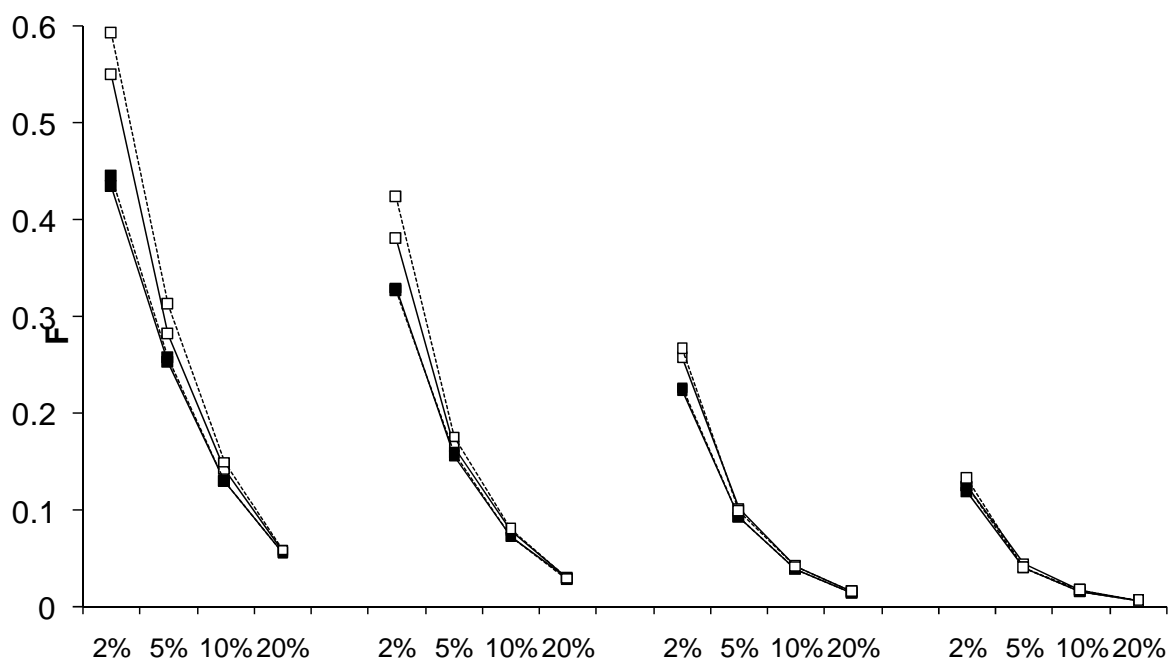

$h^{2}=0.5$

$2 \% \quad 5 \% 10 \% 20 \% \quad 2 \% \quad 5 \% 10 \% 20 \%$

$2 \% \quad 5 \% 10 \% 20 \%$

$2 \% \quad 5 \% \quad 10 \% 20 \%$

$\%$ of selected animals
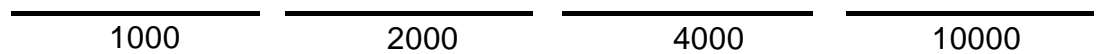

Population size

b

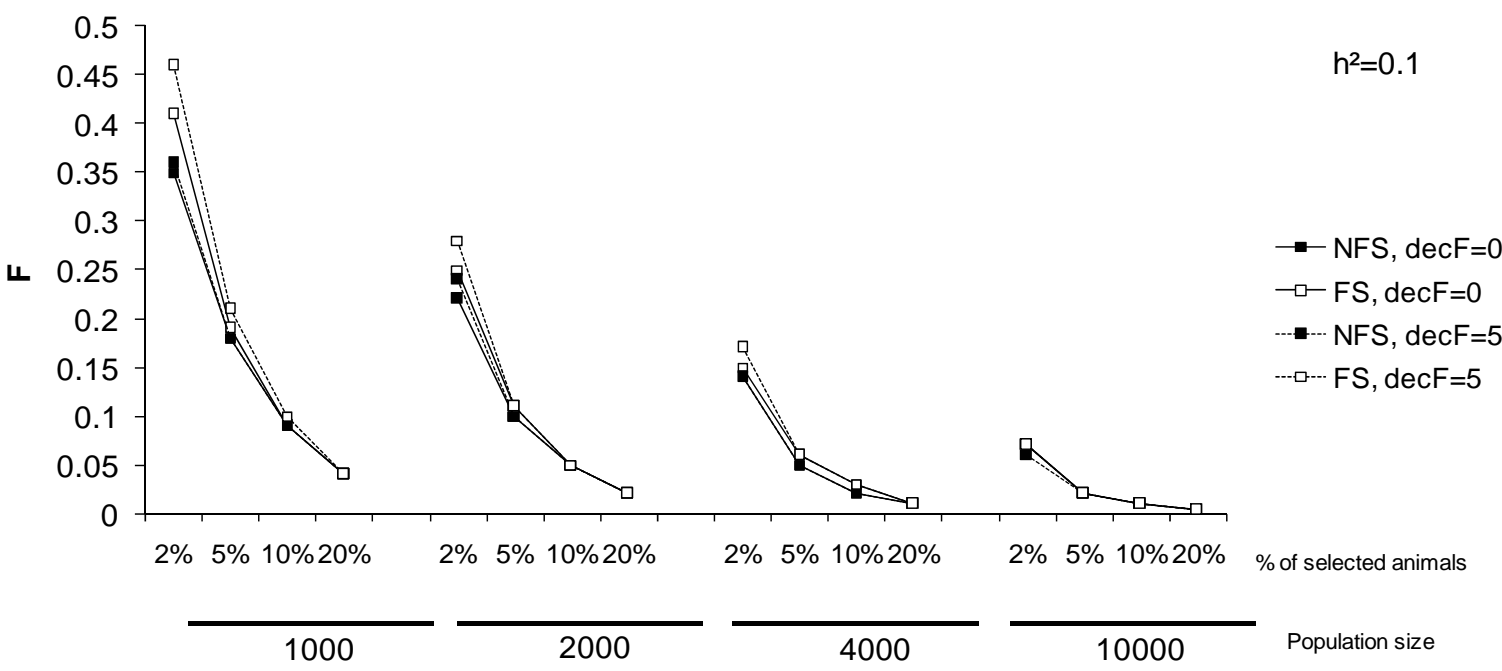


Table 1. Standard errors of phenotypic means and significance $(P)$ of the test for a difference between the phenotypic means when full sib matings are allowed (FS) or avoided (NFS). Bold figures correspond to significant differences $(P<0.05)$

\begin{tabular}{|c|c|c|c|c|c|c|c|c|}
\hline Heritability & $\begin{array}{l}\text { Population } \\
\text { size } \\
\text { before } \\
\text { selection }\end{array}$ & $\begin{array}{c}\text { Proportion } \\
\text { of } \\
\text { selected } \\
\text { animals } \\
(\%) \\
\end{array}$ & $\begin{array}{c}\mathrm{NFS} \\
\operatorname{decF}=0\end{array}$ & $\begin{array}{c}F S \\
\operatorname{dec} F=0\end{array}$ & $\begin{array}{c}P \\
\operatorname{decF}=0\end{array}$ & $\begin{array}{c}\text { NFS } \\
\text { decF=5 }\end{array}$ & $\begin{array}{c}F S \\
\operatorname{decF}=5\end{array}$ & $\begin{array}{c}P \\
\text { decF=5 }\end{array}$ \\
\hline \multirow{16}{*}{0.5} & \multirow{4}{*}{1000} & 2 & - & 2.39 & - & - & 3.37 & \\
\hline & & 5 & 1.38 & 1.54 & 0.10 & 1.75 & 1.86 & $5.7^{\mathrm{E}}-13$ \\
\hline & & 10 & 1.21 & 1.19 & 0.36 & 1.25 & 1.48 & 0.003 \\
\hline & & 20 & 0.96 & 0.91 & 0.87 & 0.85 & 0.85 & 0.09 \\
\hline & \multirow{4}{*}{2000} & 2 & 1.58 & 2.24 & 0.002 & - & 2.78 & $2.6^{\mathrm{E}}-20$ \\
\hline & & 5 & 1.09 & 1.20 & 0.01 & 1.2 & 1.31 & 0.0003 \\
\hline & & 10 & 0.84 & 0.93 & 0.18 & 0.91 & 0.65 & 0.003 \\
\hline & & 20 & 0.66 & 0.66 & 0.55 & 0.62 & 0.55 & 0.61 \\
\hline & \multirow{4}{*}{4000} & 2 & 1.31 & 1.75 & 0.0005 & 1.32 & 2 & $1.78^{\mathrm{E}}-8$ \\
\hline & & 5 & 0.99 & 0.92 & 0.92 & 0.92 & 0.81 & 0.003 \\
\hline & & 10 & 0.66 & 0.71 & 0.51 & 0.69 & 0.65 & 0.019 \\
\hline & & 20 & 0.45 & 0.5 & 0.25 & 0.5 & 0.47 & 0.76 \\
\hline & \multirow{4}{*}{10000} & 2 & 1.02 & 1.03 & 0.05 & 0.97 & 0.98 & $2.17^{E}-5$ \\
\hline & & 5 & 0.59 & 0.62 & 0.14 & 0.61 & 0.59 & 0.07 \\
\hline & & 10 & 0.44 & 0.44 & 0.50 & 0.42 & 0.4 & 0.07 \\
\hline & & 20 & 0.22 & 0.24 & 0.83 & 0.26 & 0.24 & 0.32 \\
\hline \multirow{16}{*}{0.1} & \multirow{4}{*}{1000} & 2 & 1.04 & 1.24 & 0.93 & 1.09 & 1.85 & $5.6 \mathrm{E}-13$ \\
\hline & & 5 & 0.88 & 0.9 & 0.18 & 0.62 & 1.08 & $2.9 \mathrm{E}-5$ \\
\hline & & 10 & 0.59 & 0.71 & 0.54 & 0.6 & 0.56 & 0.03 \\
\hline & & 20 & 0.45 & 0.45 & 0.91 & 0.36 & 0.35 & 0.03 \\
\hline & \multirow{4}{*}{2000} & 2 & 0.87 & 0.95 & 0.13 & 0.84 & 1.33 & $1.2 \mathrm{E}-5$ \\
\hline & & 5 & 0.65 & 0.84 & 0.13 & 0.55 & 0.59 & 0.0001 \\
\hline & & 10 & 0.45 & 0.47 & 0.04 & 0.48 & 0.45 & 0.0004 \\
\hline & & 20 & 0.3 & 0.3 & 0.87 & 0.33 & 0.35 & 0.56 \\
\hline & \multirow{4}{*}{4000} & 2 & 0.81 & 0.68 & 0.79 & 0.69 & 0.7 & 2.3E-5 \\
\hline & & 5 & 0.46 & 0.47 & 0.03 & 0.4 & 0.46 & 0.64 \\
\hline & & 10 & 0.33 & 0.39 & 0.10 & 0.36 & 0.28 & 0.77 \\
\hline & & 20 & 0.17 & 0.2 & 0.79 & 0.28 & 0.22 & 0.70 \\
\hline & \multirow{4}{*}{10000} & 2 & 0.48 & 0.49 & 0.15 & 0.48 & 0.57 & 0.09 \\
\hline & & 5 & 0.35 & 0.35 & 0.47 & 0.3 & 0.28 & 1 \\
\hline & & 10 & 0.2 & 0.2 & 0.46 & 0.2 & 0.2 & 0.62 \\
\hline & & 20 & 0.14 & 0.14 & 0.16 & 0.14 & 0.17 & 0.75 \\
\hline
\end{tabular}

